> Le Japon est un des pays les plus peuplés du monde avec une densité de 337 habitants par $\mathrm{km}^{2}$. Sa population est de 127417244 habitants pour une superficie de $377835 \mathrm{~km}^{2}$. Pourtant, I'Institut national de recherche sur la population et la sécurité sociale est préoccupé : depuis 1950, la natalité ne cesse de baisser et certaines projections alarmistes prédisent la disparition complète de la population japonaise pour I'an 3000.<

\section{(1)}

Au cours de l'année 2008, plusieurs publications basées sur le recensement, qui a eu lieu en 2005, analysent données et perspectives et cherchent des solutions pour limiter la décroissance et le vieillissement de la population. Sur le site de l'Institut japonais de recherche sur la population défile, en animation, la pyramide des âges de la population japonaise de 1930 à $2055^{1}$ et, en France, un article, très complet, vient de rendre compte de la situation démographique de ce pays [1].

\title{
Démographie japonaise
}

\section{Paradoxes et perspectives}

\section{Simone Gilgenkrantz}
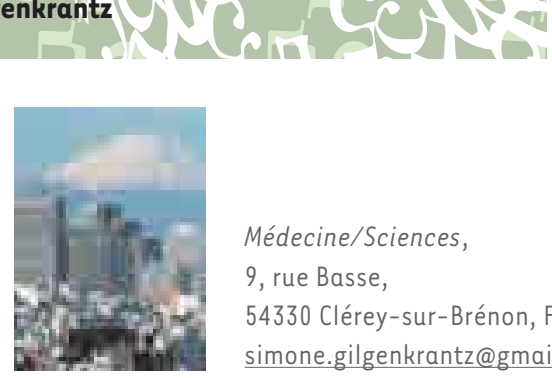

Médecine/Sciences,

9 , rue Basse,

54330 Clérey-sur-Brénon, France.

simone.gilgenkrantz@gmail.com

durant les années de guerre. La fécondité par femme commence à baisser: elle est alors de 3, 65 et l'avortement est autorisé depuis 1948. Le taux de mortalité infantile a diminué de moitié: de 124 pour 1000 naissances, il est passé à $60 \%$. Avec $4,9 \%$ au lieu de $4,8 \%$ en 1930 , le pourcentage des plus de 65 ans n'a guère augmenté. On note cependant un net allongement de la durée de vie, avec une espérance de vie à la naissance de 59,57 années pour les hommes et de 62,97 pour les femmes. Les premiers centenaires font leur apparition et font l'objet d'une attention respectueuse (Figure IB).

En 1980, l'aspect de la figure a pris une configuration étrange (Figure IC). En plus des stigmates de la guerre, on observe, comme dans presque tous les pays, une augmentation de la population correspondant au baby boom. Mais celui-ci n'a duré au Japon que trois ans environ (1947-49). Puis on remarque une diminution importante du nombre des naissances pour l'année 1966. Aussi étrange que cela paraisse pour des

\section{Histoire du Japon et pyramide des âges}

En 1930, la population totale du Japon est de 64450000 habitants; l'espérance de vie à la naissance est de 44,82 années pour les hommes et de 46,54 pour les femmes; la fécondité est de 4,7 enfants par femme; $4,8 \%$ seulement de la population a plus de 65 ans et il n'y a aucun centenaire. Comme dans beaucoup de pays à cette époque, la pyramide des âges correspond géométriquement parlant... à une pyramide (Figure IA). Vingt ans après, en 1950, la pyramide des âges a changé. Elle est marquée par les événements de l'histoire, avec des encoches correspondant à la diminution de la population

${ }^{1}$ http://www.ipss.go.jp/index-e.html

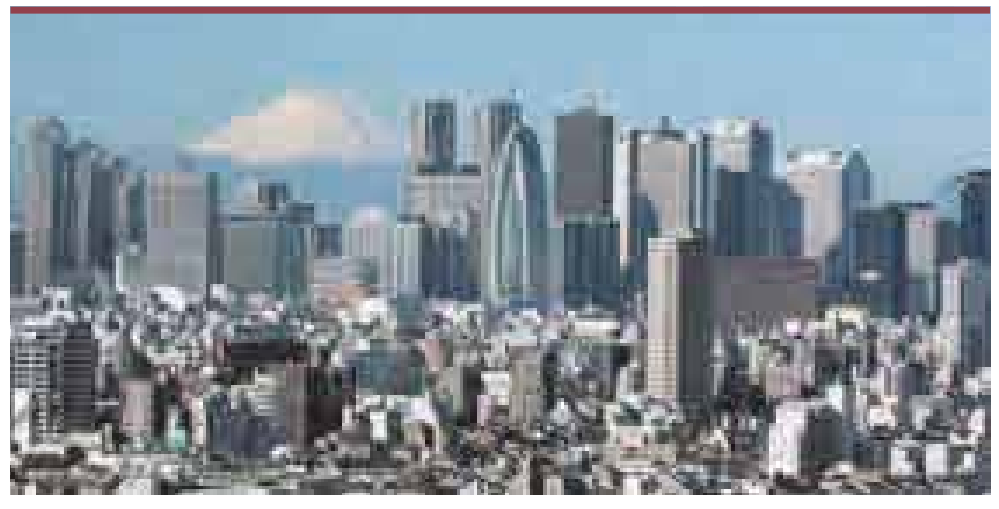

Occidentaux, elle n'est pas due à un conflit ou à une crise financière (qui survient précisément dans les années 1980 à la suite du premier choc pétrolier), mais au symbole astrologique de l'année de naissance. En effet, le zodiaque chinois comporte 12 signes animaux et se renouvelle donc tous les 12 ans. Mais s'y associent les éléments fondamentaux: l'Or, l'Eau, le Bois, le Feu et la Terre et certaines associations sont des plus funestes. Celle du Cheval et du Feu (Hinoe Uma) est une véritable malédiction car tôt ou tard les filles «cheval de feu» détruisent leurs maris moralement ou même physiquement !² C'est pourquoi le taux de natalité en 1966 a chuté de $18,7 \%$ à $13,7 \%$, avec

${ }^{2}$ Cette croyance, uniquement japonaise, reposerait sur l'histoire d'une jeune fille, Yaoya Oshichi, née en 1666 , sous le signe du Cheval de Feu, incendiaire à 16 ans, et qui aurait provoqué la mort de son ami. 


\begin{tabular}{|lcccc}
\hline \multicolumn{5}{c}{ Centenaires par sexe de $\mathbf{1 9 6 3}$ à $\mathbf{2 0 0 6}$} \\
\hline Année & Total & Hommes & Femmes & \% de femmes \\
\hline 1963 & 153 & 20 & 133 & 86,9 \\
\hline 1970 & 310 & 62 & 248 & 80 \\
\hline 1980 & 968 & 174 & 794 & 82 \\
\hline 1985 & 1740 & 359 & 1381 & 79,4 \\
\hline 1990 & 3298 & 680 & 2618 & 79,4 \\
\hline 1995 & 6378 & 1255 & 5123 & 80,3 \\
\hline 2000 & 13036 & 2158 & 10878 & 83,4 \\
\hline 2005 & 25554 & 3779 & 21775 & 85,2 \\
\hline 2006 & 28395 & 4150 & 24245 & 85,4 \\
\hline 2007 & 32295 & 4613 & 27682 & 85,7 \\
\hline
\end{tabular}

une baisse plus sensible des filles [2]. Mis à part cette particularité exclusivement nippone, l'espérance de vie s'est accrue de façon significative: 73,35 années pour les hommes à la naissance et 78,76 pour les femmes. On compte 968 centenaires dont 174 hommes et 794 femmes $(82 \%)^{3}$. Depuis 1963, ils font l'objet de statistiques spécifiques (Tableau I).

En 1990, la courbe de décroissance de la fertilité (qui est à 1,57) laisse prévoir un abaissement à 1,53 , avec la perspective d'un déclin de la population à partir de 2010. Cette constatation, jointe aux difficultés économiques rencontrées à cette époque, a créé dans le pays une secousse psychologique appelée le 1.53 shock, qui fut à l'origine d'une prise de conscience. Le gouvernement a alors effectué une grande enquête dans la population pour chercher à comprendre les raisons de cette diminution progressive de la fertilité nippone et les moyens d'y remédier.

À partir du recensement de 2002, l'Institut national de recherche sur la population et la sécurité sociale décide de scinder le journal qui rendait compte de sa double activité, études démographiques d'une part et sécurité sociale d'autre part. II crée The Japanese Journal of Population et The Japanese Journal of Social Security Policy pour mieux rendre compte de l'évolution démographique et pour analyser les projections jusqu'en 2050. Une autre revue est intitulée Journal of Population Problems.

\section{La population japonaise : constat}

De la première étude effectuée en 1992 au moment du 1.53 shock [3], il ressort que les Japonais souhaitent avoir au moins deux enfants. Plus de $40 \%$ d'entre eux considèrent que la perspective d'une diminution de la population est préoccupante. Mais ils estiment aussi que l'état n'aide pas suffisamment les familles ( $53 \%$ ), que l'environnement n'est pas favorable en particulier pour les logements (29\%) et la prise en charge des enfants (garderie et allocations scolaires) (13\%). Les femmes les plus jeunes (entre 20 et 40 ans) rejettent

\footnotetext{
${ }^{3}$ Pour leur $100^{\mathrm{e}}$ anniversaire, les Japonais reçoivent tous de l'État un gobelet d'argent, mais, par souci d'écono-
} mie, celui-ci a décidé en 2009 de réduire la taille du gobelet (le diamètre passant de 10,5 à $9 \mathrm{~cm}$ ).
Tableau I. Progression du nombre des centenaires de 1963 à 2007. Un relevé est fait en septembre de chaque année (les japonais vivant à l'étranger ne sont pas pris en compte) (source: travaux de l'Institut national de recherche sur la population et la sécurité sociale).

la façon de vivre traditionnelle (avec une famille étendue regroupant trois générations et où elles ont l'entière charge des tâches ménagères), tandis que les plus âgées souhaitent que les avortements soient soumis à une législation plus restrictive. La plupart des femmes ne sont pas favorables aux contraceptifs oraux (accusés par le gouvernement de favoriser la propagation du Sida) ; $75 \%$ des couples qui recourent à une contraception choisissent le préservatif; 20 à $30 \%$ considèrent la stérilisation comme un bon moyen contraceptif pour ceux qui ne souhaitent plus d'enfants. Enfin, 41 \% des Japonais sont favorables à une politique d'immigration plus ouverte. En 2008, l'Institut national de recherche sur la population et la sécurité sociale publie l'état des lieux en tenant compte de 25 indicateurs démographiques qui analysent non seulement les données démographiques, mais aussi les changements survenus dans les comportements des Japonais et des Japonaises [4].

Nous en évoquerons quelques-uns :

- La population a migré de la campagne vers les villes: de $37,3 \%$ en zone urbaine en 1950 , elle est passée à $78,7 \%$ en 2000. Parallèlement, les familles étendues ( $54 \%$ en 1975) ont fait place aux familles nucléaires, avec $25,6 \%$ de ménages d'une seule personne, et $6,7 \%$ de femmes seules avec enfant(s).

- L'avortement, autorisé au Japon dès 1948, trois ans après la défaite, dans un pays dévasté par la Seconde Guerre mondiale, n'est pas le fruit de mouvements féministes, mais d'une loi, the eugenic protection law, qui a été révisée en 1996 pour devenir the maternal body protection law. Le recours à l'avortement a été largement utilisé dans les années 1950 par des femmes mariées pour des raisons «économiques » et son taux reste encore élevé actuellement (28,7 pour mille femmes en 2000). - La contraception en revanche s'est développée lentement. Le recours à la pilule contraceptive semble avoir rencontré des réticences de tous ordres mais elle est actuellement remboursée par la sécurité sociale. En 2000, 55,9\% des femmes en âge de procréer déclarent avoir recours à un moyen contraceptif. - Le mariage, qui allait de soi pour toutes les femmes, survient de plus en plus tard: en 1930, 87,6\% des femmes étaient mariées à 30 ans, en 2000 ce pourcentage n'est plus que de $43,5 \%$. II ne semble plus indispensable puisque $23,7 \%$ des femmes ne se marient pas, bien que $2 \%$ seulement se déclarent hostiles au mariage. Le divorce est plus fréquent. En 2000 , il est de 2,2 pour 1000 couples. Le problème des pensions alimentaires pour les femmes divorcées avec enfant(s) semble s'être amélioré récemment à la suite d'une modification de la législation. 


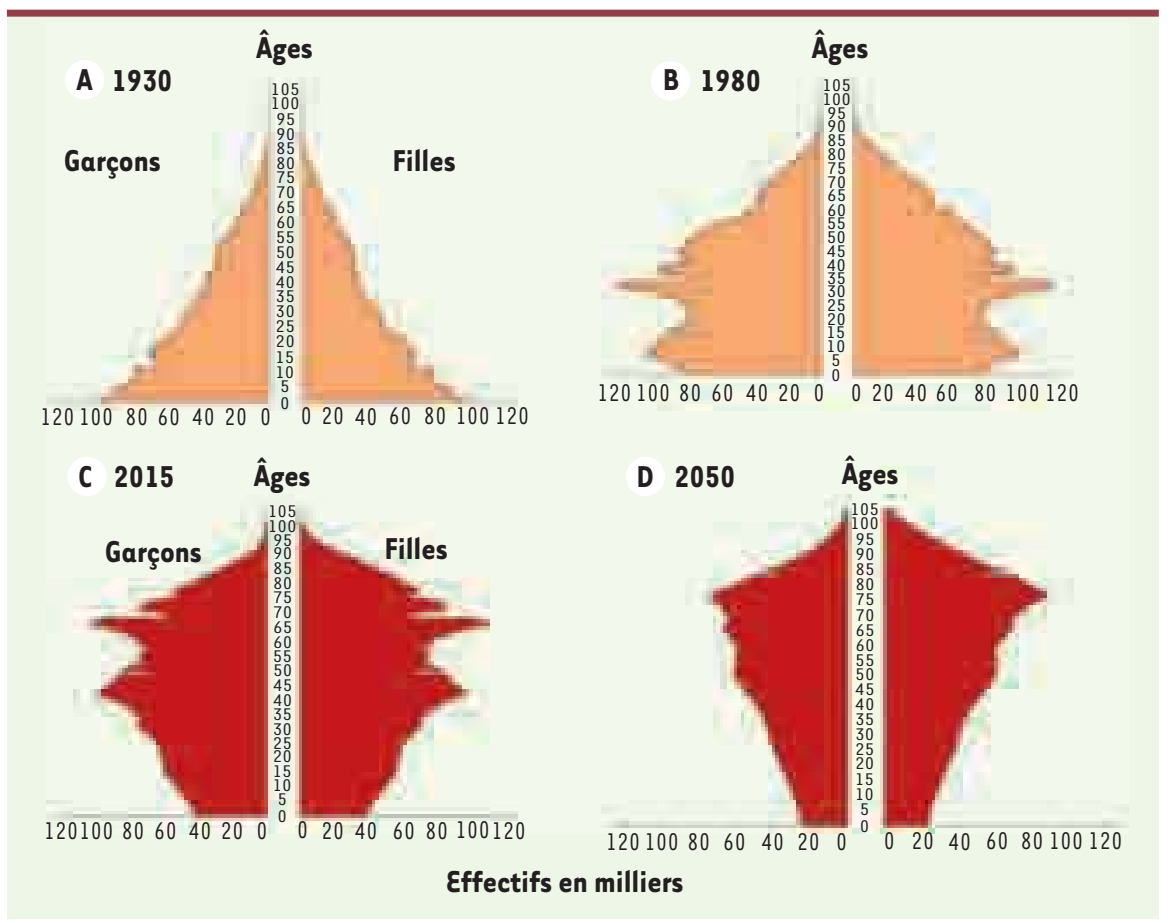

Figure 1. La pyramide des âges au Japon. En 1930 (A), 1980 (B) (en orange), et les projections pour 2015 (C) et 2050 (D) (en rouge). On constate la diminution due à l'année du cheval de feu (1966) et aux années de guerre (1940-1944) ainsi que l'allongement de la durée de vie et la diminution des naissances. Données provenant du site de l'Institut national de recherche sur la population et la sécurité sociale (http://www.ipss.go.jp/index-e.html).

(Figure 2A). En raison de la décroissance de la population, dans le meilleur des cas, la population japonaise serait légèrement inférieure à 100 millions d'habitants en 2055 (Figure 2B). Quant à l'espérance de vie moyenne, en 2055, si les courbes continuent leur trajectoire, elle serait aux alentours de 90 ans pour les femmes et de 83 ans pour les hommes (Figure 2C). Enfin le pourcentage de personnes âgées dans la population générale a été prolongé jusqu'en 2105. Les trois paramètres de la natalité ont été combinés avec les trois paramètres de l'al-

- La moyenne d'âge au premier mariage n'a pas changé pour les hommes (aux alentours de 28 ans), mais pour les femmes, elle est passée de 23 ans en 1930 à 27 ans en 2000 .

- La naissance du premier enfant est plus tardive (28,5 ans en 2007 contre 25,1 dans les années 1950). L'âge moyen de procréation est passé de 28,9 ans en 1955 à 29,9 ans en 2004 .

\section{La population japonaise : projections et conclusions}

Devant ce constat et les perspectives qu'il laisse entrevoir pour les prochaines décennies, I'Institut national de recherche sur la population et la sécurité sociale, responsable à la fois de l'analyse des études démographiques et du poids des charges sociales, ne peut qu'être préoccupé. Car la baisse de la natalité associée à l'allongement de la durée de la vie va encore augmenter le taux de dépendance démographique ${ }^{4}$. Avec le vieillissement des personnes nées pendant le baby boom, la population active (comprise entre 15 et 64 ans) va diminuer malgré l'augmentation du travail des femmes. De plus, à partir de 2001 , les offres de travail se sont réduites. L'augmentation prévisible du chômage risque de limiter le travail des plus de 64 ans qui étaient jadis nombreux à conserver une activité rémunérée.

Il semble que I'Institut ait repris à son compte la formule d'Émile de Girardin «Gouverner, c'est prévoir», car dans une publication faite en 2008 à partir des courbes obtenues de 1955 à 2005, des projections ont été faites pour différents paramètres avec trois hypothèses : basse, moyenne ou haute [5].

Concernant la natalité, elle pourrait, soit se maintenir à 1,3 enfant par femme comme en 2005 (hypothèse moyenne), soit remonter à 1,5 (hypothèse haute)

\footnotetext{
${ }^{4}$ Rapport du nombre de personnes de moins de 15 ans et de plus de 65 ans sur le nombre d'actifs de 15 à 64 ans.
}

longement de la vie: dans le pire des cas (natalité la plus basse combinée avec l'allongement le plus élevé), le pourcentage de sujets âgés atteindrait presque $50 \%$ vers les années 2075-85! (Figure 2D).

Mais le Japon n'est pas seul dans ce cas. Des études comparables dans les pays européens montrent que certains d'entre eux, avec des taux de natalité de 1,38 à 1,34 (Allemagne, Italie, Espagne et Grèce) se trouvent dans une situation voisine avec prévision d'une diminution de la population dès 2020 [6]. Et il faut se garder de conclure car les prévisions à long terme se sont souvent avérées erronées : l'avenir n'est pas écrit [7]. $\diamond$

\section{CONFLIT D'INTÉRÊTS}

L'auteur déclare n'avoir aucun conflit d'intérêts concernant les données publiées dans cet article.

\section{SUMMARY}

Japanese demography: paradoxes and perspectives Japan is one of the most densely-populated countries with a population estimated at 127.4 millions and an average density of 337 persons per square kilometer. But the National Institute of Population and Social Security Research is concerned: since 1950, the total fertility rate is declining and some pessimistic projections predict the japanese population extinction to 3000 . During 2008, several reports, based on the major results of 2005 census, analyse population process components and establish projections based on alternative assumptions, looking for solutions limiting the population dependency ratio. On the site of the National Institute of Population and Social Security Research, the 


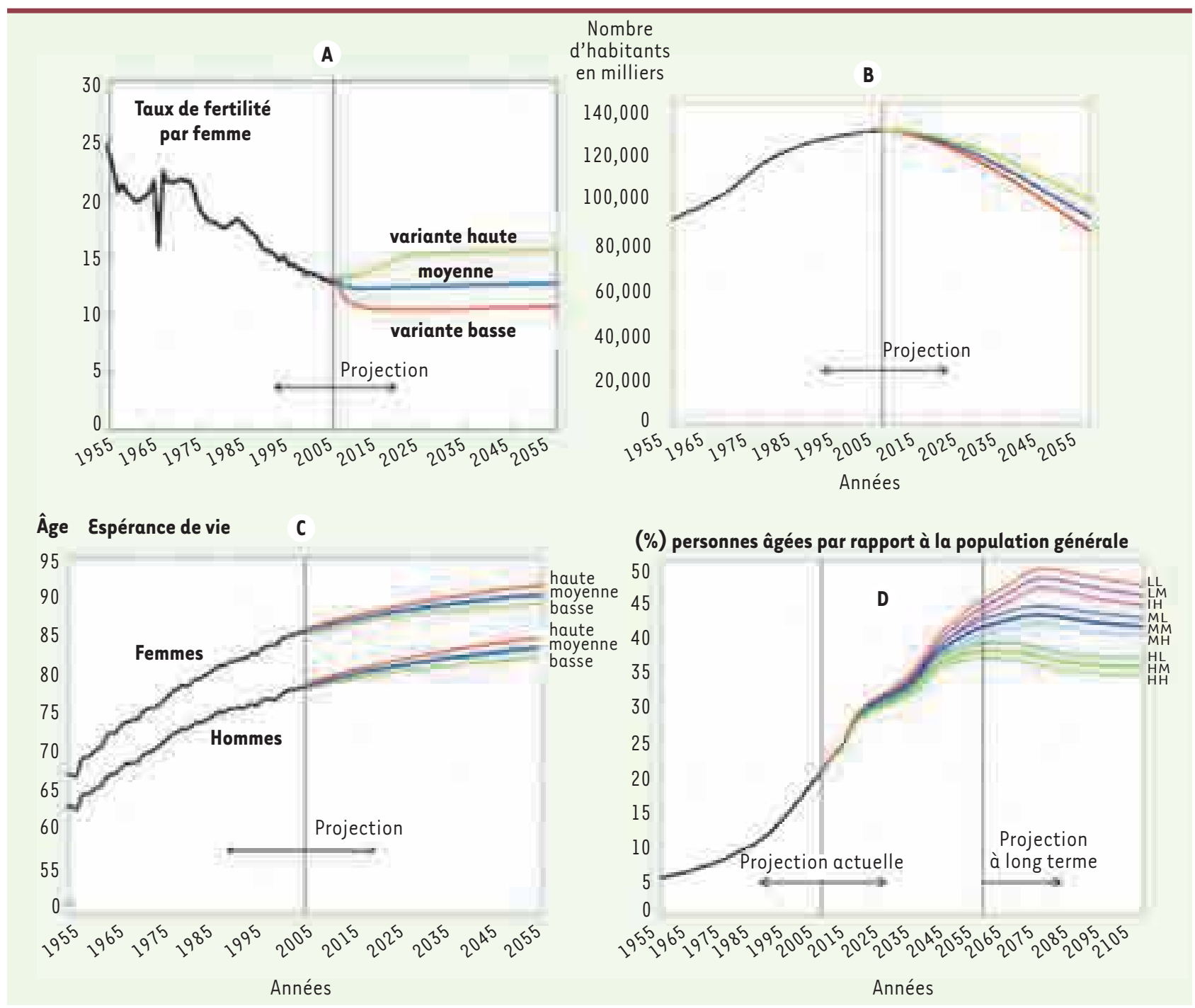

Figure 2. Courbes représentant différentes hypothèses d'évolution de la démographie japonaise. A. Fertilité par femme avec trois hypothèses en projection. B. Courbe de la population japonaise : son évolution jusqu'en 2005, avec une projection jusqu'en 2055. C. Courbe de l'espérance de vie jusqu'en 2006 avec projection jusqu'en 2055. D. Pourcentage de personnes âgées par rapport à la population générale: données actuelles, projection en 2055 , et projection à long terme avec trois variantes de natalité et trois variantes d'allongement de la durée de vie. La première lettre représente la fertilité, et la seconde la mortalité. $H$ : high; $M$ : medium; $L$ : low.

population pyramids are shown from 1930 to 2055. Recently, the French National Institute for demographic studies gave a comprehensive report on the Japanese demographic status, explaining the possible consequences for the country's demographic future. $\diamond$

\section{RÉFÉRENCES}

1. Véron J. Le Japon face au déclin annoncé de sa population. Populations Sociétés octobre $2008, n^{\circ} 449$.

2. Biraben JN. L'année cheval de feu. Population $1968 ; n^{\circ} 1: 154-62$

3. Yanagishita M. Japan's declining fertility: 1.53 shock. Popul Today $1992 ; 20: 3-4$.

4. McDonald P. Very low fertility consequences, causes and policy approaches. Japan J Popul $2008 ; 6: 19-23$

5. Kaneko R, Ishikawa A, Ishii F, et al. Populations projections for Japan : 2006-2055, outline of results, methods, and assumptions. Japan J Popul 2008 6: 76-114.
6. http://www.berlin-institut.org

7. Jacquard A, Kahn A. L'avenir n'est pas écrit. Paris : Bayard Centurion, 2001, 254 p.

\section{NOTE AJOUTÉદ AUX ÉPREUVES}

Dans les projets du Parti Démocrate qui vient de gagner les élections, plusieurs mesures sont proposées pour lutter contre la dénatalité, entre autres la création d'une allocation mensuelle de 26000 Yens, soit $200 €$ pour chaque enfant.
TIRÉS À PART

S. Gilgenkrantz 